\title{
Pneumatosis cystoides coli
}
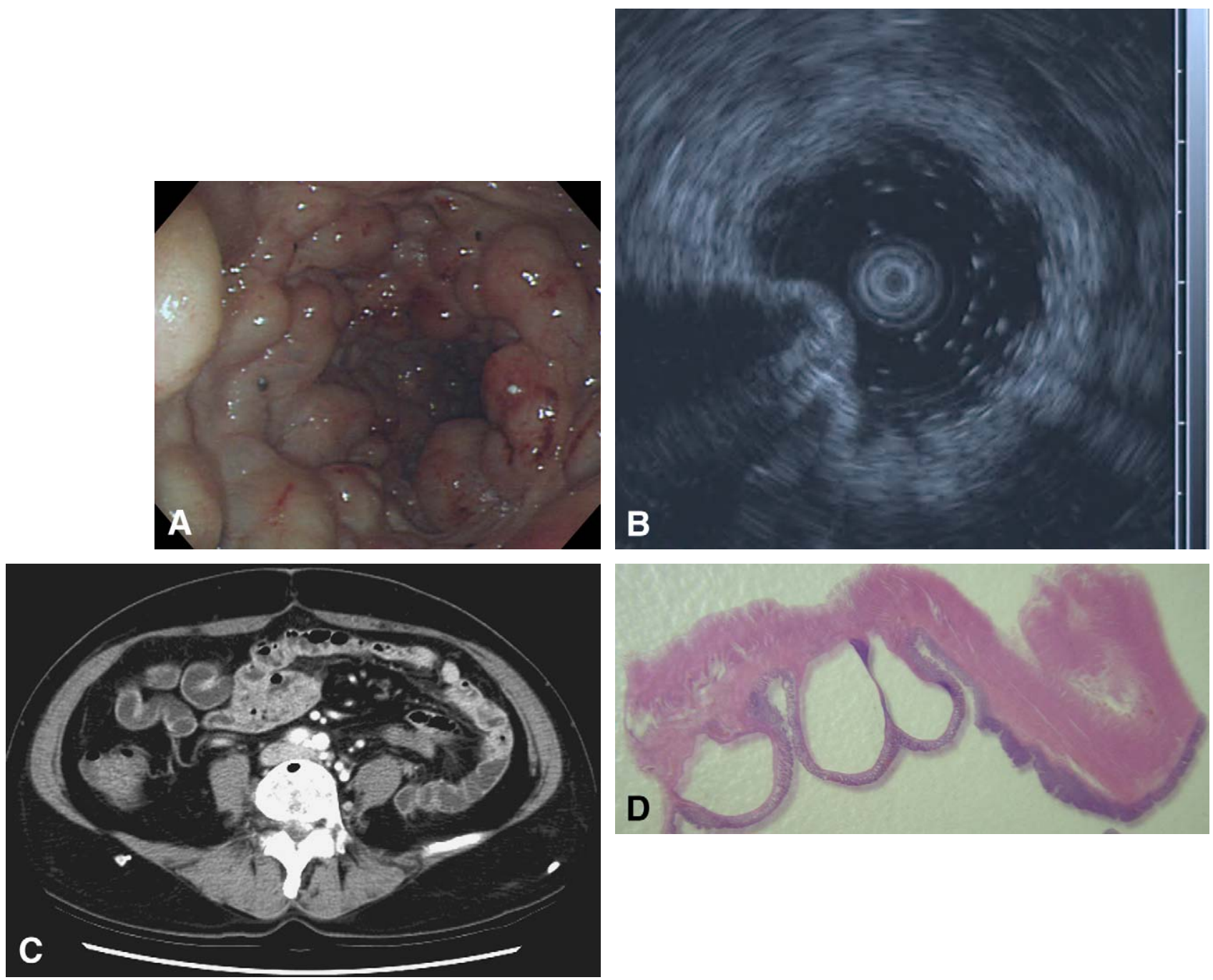

A 66-year-old woman with hypertension had had postprandial abdominal distention and discomfort during defecation for 1 year. Colonoscopy revealed multiple 0.5 to $3 \mathrm{~cm}$ polypoid grape-like masses with erythematous mucosa 20 to $40 \mathrm{~cm}$ above the anal verge (A). EUS with an ultrasonic probe (UM-DP12-25R, 12MHz, Olympus Optical, Tokyo, Japan) passed through the working channel of the colonoscope showed multiple anechoic lesions with air inside at the submucosal layer of the descending colon (B). CT showed clusters of air pockets in the wall of the colon (C), supporting the diagnosis of pneumatosis cystoides coli. The patient underwent left hemicolectomy. Pathologic examination revealed many submucosal cystic spaces with thin fibrous walls lined by flattened cells (D, H\&E, orig. mag. $\times 3$ ). The overlying colonic mucosa showed decreased thickness and focal erosive changes; the remainder of the colonic mucosa was unremarkable. The patient has remained asymptomatic after operation.

\section{DISCLOSURE}

None of the authors have any disclosures to make.

Yu-Ting Chang, MD, MS, Ming-Chu Chang, MD, Shu-Chen Wei, MD, PhD, Jau-Min Wong, MD, PhD, Departments of Internal Medicine National Taiwan University Hospital Taipei, Taiwan

doi:10.1016/j.gie.2006.05.012 


\section{Commentary}

Pneumatosis intestinalis is of two types: cystoides and linearis. The former is seen with a wide variety of conditions (eg, pulmonary, peptic, collagen vascular, malignant, and postprocedural) and may affect small intestine or colon; the latter is seen as a consequence of necrotic bowel (thus mandating surgery) or in patients with acquired immunodeficiency syndrome complicated by opportunistic infections (eg, cryptosporidium and cytomegalovirus) in whom it may be reversible if the underlying infection is controlled. Pneumatosis is usually asymptomatic but may cause diarrhea, constipation, abdominal pain, and bleeding and may be complicated by obstruction (adhesions, volvulus, intussusception) and benign pneumoperitoneum. Because of technical advances in imaging, these gas-filled cysts are no longer confused with colon polyps and "thumbprinting" as they had been on plain film and barium enema examinations. This case shows how current technology beautifully enables their definitive diagnosis and, in this unusual case, the need for surgical resection of the pneumatosis cyst-bearing segment of bowel.

Lawrence J. Brandt, MD Associate Editor for Focal Points 